\title{
On the bit-size of non-radical triangular sets
}

\author{
Xavier Dahan* \\ Ochanomizu university, Faculty of General Educational Research \\ dahan.xavier@ocha.ac.jp
}

\begin{abstract}
We present upper bounds on the bit-size of coefficients of non-radical lexicographical Gröbner bases in purely triangular form (triangular sets) of dimension zero. This extends a previous work 4], constrained to radical triangular sets; it follows the same technical steps, based on interpolation. However, key notion of height of varieties is not available for points with multiplicities; therefore the bounds obtained are less universal and depend on input data. We also introduce a related family of non-monic polynomials that have smaller coefficients, and smaller bounds. It is not obvious to compute them from the initial triangular set though.
\end{abstract}

\section{Introduction}

Triangular sets are the core objects of the triangular decomposition methods to solve polynomial systems [6]1/7/21]. Algorithms in this realm are somewhat based on the generalization of algorithms for univariate polynomials to multivariate ones, yielding some splittings viz. "decomposition". The outputs are most of the time regular chains (a.k.a regular sets). In dimension zero they can be made particularly simple: they form a reduced lexicographic Gröbner basis $\left(t_{1}\left(x_{1}\right), t_{2}\left(x_{1}, x_{2}\right), \ldots, t_{n}\left(x_{1}, \ldots, x_{n}\right)\right)$. We will refer to such a family as a triangular set in this article.

To solve polynomial systems, it is enough to represent the radical ideal generated by the input polynomials, thereby most previous works focus on radical triangular sets. However, triangular sets have the ability to represent some nonradical ideals (called thereafter triangular ideals); Moreover the radical of the ideal generated by a triangular set is not necessarily triangular, requiring extra work to decompose it into triangular sets. If we compare with the Rational Univariate Representation (RUR, see [10]), only the multiplicity (which is just a number) of a root is given. Therefore beyond the theoretical interest, it is worth studying non-radical triangular sets.

In this article we unveil the structure and prove upper bounds on the bit-size of coefficients of such triangular sets. This is an attempt of generalization from radical to non-radical triangular sets, of the results given in 44. Let us recall briefly the strategy of this paper, since we will follow it.

Step 1) Given the solution points, some interpolation formulas are proved to reconstruct the triangular set from the points.

* work supported by the JSPS grant Wakate B No. 50567518 
Step 2) These formula allows to control the growth of coefficients in function of that of the points.

Step 3) A tool from Diophantine geometry called height of variety defined through a Chow form is introduced. It measures somewhat the arithmetic complexity of the variety, and is endowed of an arithmetic analogue of the Bézout theorem (degree of intersection). This "Arithmetic Bézout theorem" provides upper bounds in function of any input polynomial system.

Step 4) A simple modification of the interpolation formulas, called barycentric form of Lagrange interpolation defines a family of non-monic polynomials which have smaller coefficients.

We present extensions of Steps 1)-2), and partially 4), to non-radical triangular sets. As for Step 3) the tool (height of variety) is not available for multiple points. While interpolation in [4] is multivariate Lagrange, here it is more general and encompasses multivariate Hermite interpolation. The input data are not points but primary ideals assumed to be given by a triangular set (see (10)) The related family of non-monic polynomials mentioned in Step 4) and denoted $N_{\ell}$ are defined in Theorem 1. In comparison with [4, they seem not easy to compute from the triangular set $T$ (see [12]) for an attempt in two variables).

Related work. For the bit-size bounds, a selection of related work concerned with the RUR, triangular sets, and lexicographic Gröbner bases is [8/4319. The bounds presented are the first ones dealing with non-radical systems having a general type of singularities. Comparatively, a RUR can represent multiplicities (recall that this is just a number) but not a full singularity type.

Notation. $k$ will denote any field $\mathbb{Q} \subseteq k \subsetneq \overline{\mathbb{Q}}$. A polynomial ring over $k$, in $n$ variables $x_{1}, \ldots, x_{n}$ implicitly ordered such as $x_{1} \prec x_{2} \prec \cdots \prec x_{n}$, a triangular set $T=\left(T_{1}\left(x_{1}\right), \ldots, T_{n}\left(x_{1}, \ldots, x_{n}\right)\right)$ with $\operatorname{deg}_{x_{j}}\left(T_{j}\right)=d_{j}$. Its set of zeros in $\bar{k}^{n}$ is denoted $V$. For a subset $\mathcal{S}$ of an arbitrary Cartesian product $E^{m}, \mathcal{S}_{\leq \ell}$ will denote the projection of $\mathcal{S}$ on the first $\ell$ coordinates.

\section{Interpolation formula}

Input data. In the radical case, we want to interpolate points. Here, the raw input data are primary ideals associated to each solution point in $\bar{k}^{n}$. Thanks to Theorem 2.4 of [5] the primary ideals of a triangular set are triangular: the lexicographic Gröbner basis of these primary ideals are triangular sets. Still over $\bar{k}$, a prime ideal associated with $\langle T\rangle$ is of the form $\left\langle x_{1}-\alpha_{1}, \ldots, x_{n}-\alpha_{n}\right\rangle$, for a solution point $\left(\alpha_{1}, \ldots, \alpha_{n}\right)$. The corresponding primary ideal $\left\langle t^{(\alpha)}\right\rangle$ has the following shape (Proposition 2.2 of [5]): First $t_{1}^{(\alpha)}\left(x_{1}\right)=\left(x_{1}-\alpha_{1}\right)^{\delta_{1}(\alpha)}$ and in general $t_{n}^{(\alpha)}\left(x_{1}, \ldots, x_{n}\right)$ is equal to:

$$
\left(x_{n}-\alpha_{n}\right)^{\delta_{n}(\alpha)}+\sum_{i_{1}=0}^{\delta_{1}\left(\alpha_{1}\right)-1} \sum_{i_{n-1}=0}^{\delta_{n-1}(\alpha)-1} \sum_{i_{n}=0}^{\delta_{n}(\alpha)-1} c_{\alpha}\left[i_{1}, \ldots, i_{n}\right] \prod_{j=1}^{n}\left(x_{j}-\alpha_{j}\right)^{i_{j}}
$$

where: (i) For $1 \leq u \leq n, \operatorname{deg}_{x_{u}}\left(c\left[i_{1}, \ldots, i_{n}\right]\right)<\delta_{u}(\alpha)$, 
(ii) $c\left[0, \ldots, 0, i_{\ell}\right]=0$ for all $i_{\ell}<\delta_{\ell}(\alpha)$ and for $\ell=2, \ldots, n$.

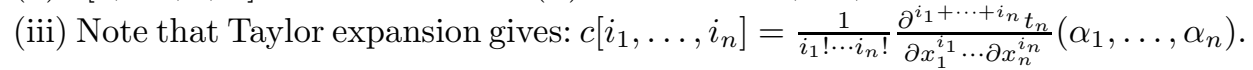

We denote by $T_{\ell+1}[\alpha]$ the polynomial $T_{\ell+1} \bmod \left\langle t_{<\ell}^{(\alpha)}\right\rangle$. Theorem 3.1 of [5], the ring $\bar{k}\left[x_{1}, \ldots, x_{n}\right] /\left\langle t_{\leq \ell}^{(\alpha)}\right\rangle$ is Henselian. Hence $T_{\ell+1}[\alpha]$ admits a unique factorization as follows:

$$
T_{\ell+1}[\alpha] \equiv \prod_{\beta \in V_{\leq \ell+1}} t_{\ell+1}^{(\beta)} \bmod \left\langle t_{\leq \ell}^{(\alpha)}\right\rangle, \text { where }\left(\beta_{1}, \ldots, \beta_{\ell}\right)=\left(\alpha_{1}, \ldots, \alpha_{\ell}\right) .
$$

and $t_{\ell+1}^{(\beta)}=\left(x_{\ell+1}-\beta_{\ell+1}\right)^{\delta_{\ell+1}(\beta)}+\sum_{i_{1}, \cdots, i_{\ell}, r} c_{\beta}\left[i_{1}, \ldots, i_{\ell}, r\right]\left(x_{\ell+1}-\beta_{\ell+1}\right)^{r} \cdot \prod_{j=1}^{\ell}\left(x_{j}-\right.$ $\left.\alpha_{j}\right)^{i_{j}}$ for some $c_{\beta}\left[i_{1}, \ldots, i_{\ell}, r\right] \in \bar{k}$. This key result allows to prove Proposition1. Notation. A sequence $\alpha^{1} \in V_{\leq 1}, \quad \alpha^{2} \in V_{\leq 2}, \ldots, \quad \alpha^{\ell} \in V_{\leq \ell}$ will not denote " $j$-th power of $\alpha$ ", but points $\alpha^{j}=\left(\alpha_{1}^{j}, \ldots, \alpha_{j}^{j}\right)$ with the additional convention that $\left(\alpha_{1}^{j}, \ldots, \alpha_{j}^{j}\right)=\left(\alpha_{1}^{j+1}, \ldots, \alpha_{j}^{j+1}\right)$. We say that $\alpha^{j+1}$ extends $\alpha^{j}$.

Proposition 1 Let $\gamma \in V_{\leq \ell+1}$ be a root that extends $\alpha=\left(\alpha_{1}, \ldots, \alpha_{\ell}\right) \in V_{\leq \ell}$.

1. $e_{\ell+1}(\gamma) \equiv \frac{T_{\ell+1}[\alpha]}{t_{\ell+1}^{(\gamma)}} \bmod \left\langle t_{\leq \ell}^{(\alpha)}\right\rangle$ is a polynomial in $\left(\bar{k}\left[x_{1}, \ldots, x_{\ell}\right] /\left\langle t_{\leq \ell}^{(\alpha)}\right\rangle\right)\left[x_{\ell+1}\right]$

2. Orthogonality: Given $\beta \neq \gamma \in V_{\leq \ell+1}$ :

$$
e_{\ell+1}(\beta) \cdot e_{\ell+1}(\gamma)=0 \text { in } A_{\alpha}:=\bar{k}\left[x_{1}, \ldots, x_{\ell+1}\right] /\left\langle t_{1}^{(\alpha)}, \ldots, t_{\ell}^{(\alpha)}, T_{\ell+1}[\alpha]\right\rangle .
$$

3. $e_{\ell+1}(\beta) \equiv 0 \bmod \left\langle t_{\leq \ell+1}^{\left(\beta^{\prime}\right)}\right\rangle$ if $\beta^{\prime} \neq \beta, \quad$ and $\widetilde{e_{\ell+1}}(\gamma) \equiv 1 \bmod \left\langle t_{\leq \ell+1}^{(\gamma)}\right\rangle$.

4. There are polynomials $u_{\ell+1}(\gamma)$ and $v$ such that

$u_{\ell+1}(\gamma) e_{\ell+1}(\gamma)+v t_{\ell+1}^{(\gamma)} \equiv 1 \bmod \left\langle t_{\leq \ell}^{(\alpha)}\right\rangle$, with $\operatorname{deg}_{x_{\ell+1}}\left(u_{\ell+1}(\gamma)\right)<\operatorname{deg}_{x_{\ell+1}}\left(t_{\ell+1}^{(\gamma)}\right)$. Denote $\widetilde{e_{\ell+1}}(\gamma) \equiv u_{\ell+1}(\gamma) e_{\ell+1}(\gamma) \bmod \left\langle t_{\leq \ell}^{(\alpha)}\right\rangle$. The family $\left\{\widetilde{e_{\ell+1}}(\gamma)\right\}_{\gamma}$ is a complete family of orthogonal idempotents of the algebra $A_{\alpha}$.

Have in mind that Lagrange interpolation use idempotents.

Theorem 1 Write $t_{\leq \ell}^{(\alpha)}=\left(t_{1}^{(\alpha)}, \ldots, t_{\ell}^{(\alpha)}\right)$ the triangular sets defining the primary ideal of associated prime $\left\langle x_{1}-\alpha_{1}, \ldots, x_{\ell+1}-\alpha_{\ell+1}\right\rangle$.

$$
T_{\ell+1} \equiv \sum_{\alpha^{1} \in V_{\leq 1}} \sum_{\alpha^{2} \in V_{\leq 2}} \cdots \sum_{\alpha^{\ell} \in V_{\leq \ell}} \widetilde{e_{1}}\left(\alpha^{1}\right) \cdots \widetilde{e_{\ell}}\left(\alpha^{\ell}\right) \cdot T_{\ell+1}\left[\alpha^{\ell}\right] \bmod \left\langle t_{\leq \ell}^{(\alpha)}\right\rangle .
$$

where it is assumed that $\alpha^{j+1} \in V_{\leq j+1}$ extends $\alpha^{j} \in V_{\leq j}$. We define $N_{\ell+1}$ by the same formula, using polynomials $e_{i}\left(\alpha^{i}\right)$ instead of $\widetilde{e_{i}}\left(\alpha^{i}\right)$.

Since the family of idempotents used to define $T_{\ell+1}$ is complete, $T_{\ell+1}$ is monic. This is not the case for $N_{\ell+1}$. Therefore they cannot be used to perform reduction through a division, but their interest lies in their small coefficients, and in that they generate the same ideal as $\left\langle T_{1}, \ldots, T_{\ell}\right\rangle$ hence encodes the same information; In the radical case they were used in conjunction of modular methods.

A natural question is whether we can compute the polynomials $N_{\ell}$ 's from the $T_{\ell}$ 's. The answer is not trivial and not addressed in these pages. However it is not unreasonable to expect an almost linear complexity algorithm to compute it, as shown for the case of two variables in [12. It boils down to compute the polynomial denoted $F_{\ell+1}$ hereunder 
Proposition 2 We have $F_{\ell+1} T_{\ell+1} \equiv N_{\ell+1} \bmod \left\langle T_{1}, \ldots, T_{\ell}\right\rangle$, with:

$$
F_{\ell+1}:=\sum_{\alpha^{1} \in V_{\leq 1}} \sum_{\alpha^{2} \in V_{\leq 2}} \cdots \sum_{\alpha^{\ell} \in V_{\leq \ell}} e_{1}\left(\alpha^{1}\right) \cdots e_{\ell}\left(\alpha^{\ell}\right)
$$

and where the same convention on $\alpha^{1}, \alpha^{2}, \ldots$ as in Theorem 1 is adopted.

In the radical case, it is easy to show that $F_{\ell}=\frac{\partial T_{1}}{\partial x_{1}} \cdots \frac{\partial T_{\ell}}{\partial x_{\ell}}$.

\section{Bit-size consideration}

Preliminary. This last section states and comments on some upper-bounds on the bit-size of coefficients in $\mathbb{Q}$ appearing in the polynomials $T$ and $N$. The difficulties compared to the radical case are first, that the interpolation formulas are more complicated to handle, and second, that there is no notion of Chow form, yet of notion height of varieties, in our non-radical context; Whereas it was a key tool in [4] to obtain intrinsic bit-size bounds (see Step 3) in Introduction). The upper bounds that we can obtain are less universal: we assume that the input primary ideals are given in triangular form, which we have written $t^{(\alpha)}$, while universal bounds would not need this assumption (another input for the primary ideals may well have smaller coefficients, hence yield better bounds). However bounds (6) give a reasonable indication on the bit-size, and are also of interest to understand the growth of coefficients in multivariate interpolation with singularities, including Hermite's as a subcase.

Statement. We use the formalism of height of polynomials classical in Diophantine approximation theory. The notation $h(f)$ denotes the height of the polynomial $f$, and can be thought as the max bit-size of its coefficients. Recall that the input "raw" data are the triangular set $\left\{t^{(\alpha)}, \alpha \in V\right\}$ generating the primary ideals of $\langle T\rangle$. With the notations of (1), this includes the exponents $\delta_{i}(\alpha)$ and coefficients $c_{\alpha}\left[i_{1}, \ldots, i_{\ell}\right]$. We define:

$$
H_{\ell}\left(\beta^{\ell}\right):=\max _{i_{1}, \ldots, i_{\ell}} h\left(c_{\beta}\left[i_{1}, \ldots, i_{\ell}\right]\right)+i_{1} h\left(\beta_{1}\right)+\cdots+i_{\ell} h\left(\beta_{\ell}\right)
$$

and $\mathrm{L}_{\ell}(T):=\max _{\alpha \in V_{<\ell}}\left(\sum_{i=1}^{\ell} H_{i}\left(\beta^{i}\right)\right)$. Denote by $\mu_{\ell}\left(\beta^{\ell}\right):=\delta_{1}\left(\beta_{1}\right) \cdots \delta_{\ell}\left(\beta_{\ell}\right)$ the local multiplicity at $\beta^{\ell}$, and finally:

$$
H_{\ell}(T):=\sum_{\beta^{\ell} \in V_{\leq \ell}} H_{\ell}\left(\beta^{\ell}\right), \quad \mu_{\ell}(T):=\max _{\beta^{\ell} \in V_{\leq \ell}} \mu_{\ell}\left(\beta^{\ell}\right), \quad D_{\ell}:=\sum_{i \leq \ell} d_{i}
$$

The bit-sizes of any coefficient of $N_{\ell+1}$, or $T_{\ell+1}$, are lower than quantities whose dominating terms are respectively:

$$
H_{\ell+1}(T)+\widetilde{O}\left(\mathrm{~L}_{\ell}(T) \cdot D_{\ell+1} \cdot \mu_{\ell}(T)\right), \quad D_{\ell+1} H_{\ell+1}(T)+\widetilde{O}\left(\mathrm{~L}_{\ell}(T) \cdot D_{\ell+1}^{2} \cdot \mu_{\ell}(T)\right)
$$

Rationale. $\widetilde{O}($.$) is a big-Oh notation that hides any additional logarithmic$ factors. The quantity $H_{\ell}\left(\beta^{\ell}\right)$ of (4) should be thought as a generalization to primary components of the height of a projective point: coefficients and exponents in the Taylor expansion (11) are simply "naturally" taken into account. If 
the point is simple, then this quantity coincides with the "traditional" height of a point. In addition, the quantity $H_{\ell}(T)$ of (5) reflects a certain additivity of the "height" under distinct primary components; this also generalizes the additivity of the height of varieties under disjoint union. Therefore, the quantities involved are "natural" extension to those more traditional used in the case of simple points. The occurrence of quantities like $\mathrm{L}_{\ell}(T)$ or the multiplicity at a point $\mu_{\ell}\left(\beta^{\ell}\right)$ is due to technical details due to the presence of multiplicities.

If $T$ generates a radical ideal, then $\mu_{\ell}\left(\beta^{\ell}\right)=1, H_{\ell}\left(\beta^{\ell}\right)=h\left(\beta^{\ell}\right)$; moreover $H_{\ell}(T) \approx h\left(V_{\leq \ell}\right), D_{\ell} \leq \operatorname{deg}\left(V_{\leq \ell}\right)$ which are respectively the height of the variety $V_{\leq \ell}$ and its degree, and if we discard the values $\mathrm{L}_{\ell}(T)$ then the bounds in (6) become roughly $h\left(V_{\ell}\right)+\widetilde{O}(\operatorname{deg}(V))$ for $N_{\ell+1}$, and $\operatorname{deg}\left(V_{\ell}\right) h\left(V_{\ell}\right)+\widetilde{O}\left(\operatorname{deg}\left(V_{\leq \ell}\right)^{2}\right)$ for $T_{\ell+1}$. These bounds are similar to the ones obtained in 4 . This shows that the bounds (6) "faithfully" extend the ones of the radical case. The difference between the bounds for $N_{\ell+1}$ and for $T_{\ell+1}$ is roughly the factor $D_{\ell}$, and this ratio is comparable to that of [4].

Finally, experiments not reported here (but see Table 1 in 12 for some data in two variables) show that the size of polynomials $T_{\ell}$ can be dramatically larger than $N_{\ell}$ 's.

\section{References}

1. P. Aubry, D. Lazard, and M. Moreno Maza. On the theories of triangular sets. J. of Symbolic Computation, 28(1,2):45-124, 1999.

2. C. Chen and M. Moreno-Maza. Algorithms for computing triangular decomposition of polynomial systems. Journal of Symbolic Computation, 47(6):610-642, 2012.

3. X. Dahan. Size of coefficients of lexicographical Gröbner baes. In ISSAC'09, pages 117-126. ACM, 2009.

4. X. Dahan and É. Schost. Sharp estimates for triangular sets. In ISSAC 2004, pages 103-110. ACM Press, 2004.

5. Xavier Dahan. Gcd modulo a primary triangular set of dimension zero. In Proceedings of ISSAC 2017, pages 109-116, New York, NY, USA, 2017. ACM.

6. M. Moreno Maza F. Lemaire and Y. Xie. The RegularChains library.

7. É. Hubert. Notes on triangular sets and triangulation-decomposition algorithms. I. Polynomial systems. volume 2630 of Lecture Notes in Comput. Sci., pages 1-39. Springer, Berlin, 2003.

8. Angelos Mantzaflaris, Éric Schost, and Elias Tsigaridas. Sparse rational univariate representation. In ISSAC 2017, page 8, 2017.

9. Esmaeil Mehrabi and Éric Schost. A softly optimal monte carlo algorithm for solving bivariate polynomial systems over the integers. Journal of Complexity, 34:78-128, 2016.

10. F. Rouillier. Solving zero-dimensional systems through the rational univariate representation. Appl. Algebra Eng. Commun. Comput., 9(5):433-461, 1999.

11. D. Wang. Elimination Methods. Texts \& Monographs in Symbolic Computation. Springer Vienna, 2012.

12. Tetsuro Yamashita and Xavier Dahan. Bit-size reduction of triangular sets in two and three variables. In SCSS'2016, volume 39 of EPiC Series in Computing, pages 169-182, 2016. 